\title{
Análise Angiográfica e Ultra-sonográfica do Stent Liberador de Novolimus para o Tratamento de Lesões Coronárias De Novo: Resultados do Estudo EXCELLA I
}

\author{
Felipe Maia ${ }^{1}$, Alexandre Abizaid ${ }^{1}$, José Ribamar Costa Jr. ${ }^{1}$, Fausto Feres ${ }^{1}$, Ana Cristina Seixas ${ }^{1}$, \\ Rodolfo Staico ${ }^{1}$, Luiz Alberto Mattos ${ }^{1}$, Andréa Abizaid ${ }^{1}$, Ricardo A. Costa ${ }^{1}$, \\ Amanda G. M. R. Sousa ${ }^{1}$, J. Eduardo Sousa ${ }^{1}$
}

\section{RESUMO}

Introdução: A reestenose intra-stent coronário sofreu marcante redução com o surgimento dos stents liberadores de medicamentos, alcançando níveis abaixo de $10 \%$ na maioria dos cenários clínicos e angiográficos. Desde então, novos dispositivos com diferentes fármacos vêm sendo testados com a finalidade de se obter perfil de eficácia pelo menos equivalente ao dos stents farmacológicos de primeira geração $\left(\right.$ Cypher $^{\circledR}$ e Taxus $\left.^{\circledR}\right)$, mas com maior segurança tardia. Objetivo: Avaliar a segurança e a eficácia na inibição da proliferação neointimal com implante de stent liberador de novolimus. Método: Estudo unicêntrico, tipo first-in-man, com inclusão de 15 pacientes portadores de angina pectoris submetidos a implante de stent liberador de novolimus. Resultados: Análise por meio de angiografia coronária quantitativa (ACQ) e ultra-som intracoronário (USIC), realizada imediatamente após implante do stent e aos quatro meses, revelou perda tardia intra-stent de 0,15 $\pm 0,29 \mathrm{~mm}$ e no segmento, de 0,07 $\pm 0,3 \mathrm{~mm}$. O volume de obstrução intra-stent foi de $2,6 \pm 2,6 \%$. Não foram observados casos de reestenose binária. O seguimento clínico aos seis meses foi obtido em todos os pacientes, não sendo observados eventos cardíacos adversos maiores nesse período. Conclusão: $O$ novo stent liberador de novolimus mostrou-se eficaz na supressão da proliferação neo-intimal. A avaliação dos resultados tardios em grupo de pacientes mais complexos é necessária para confirmar a segurança do dispositivo.

DESCRITORES: Contenedores. Coronariopatia. Ultra-sonografia de intervenção. Angiografia coronária. Materiais revestidos biocompatíveis. Implantes de medicamento.

\section{SUMMARY}

\section{Preliminary Results from the First-In-Man Study EXCELLA}

Background: Stent restenosis suffered a marked reduction with drug-eluting stents (DES) reaching levels below 10\% in most clinical and angiographic scenarios. Since then, new devices with different drugs have been tested in order to obtain an efficiency equivalent to first generation DES $\left(\right.$ Cypher $^{\circledR}$ and Taxus $\left.{ }^{\circledR}\right)$, although with better long-term safety. Our objective was to evaluate safety and neointimal proliferation inhibition with novolimuseluting stent through clinical and angiographic endpoints. Methods: Single-center, first-in-man study, including 15 patients with angina pectoris submitted to novolimuseluting stent implantation. Results: Angiographic analysis with quantitative coronary angiography (QCA) and intravascular ultrasound (IVUS) performed immediately after stent implantation and at 4-month follow-up revealed instent and in-segment late loss of $0.15 \mathrm{~mm} \pm 0.29 \mathrm{~mm}$ and $0.07 \mathrm{~mm} \pm 0.3 \mathrm{~mm}$, respectively. In-stent volume obstruction was $2.6 \pm 2.6 \%$. No cases of binary restenosis were observed. The six-month clinical follow-up revealed no major acute cardiovascular events (MACE). Conclusion: The new novolimus-eluting stent proved to be efficient in reducing late loss and supressing neointimal proliferation. Late results in more complex group of patients are needed to confirm the safety of this new device.

DESCRIPTORS: Stents. Coronary disease. Ultrasonography, interventional. Coronary angiography. Coated materials, biocompatible. Drug implants.
${ }^{1}$ Instituto Dante Pazzanese de Cardiologia - São Paulo, SP. Correspondência: Alexandre Abizaid. Instituto Dante Pazzanese de Cardiologia. Av. Dr. Dante Pazzanese, 500 - São Paulo, SP CEP 04038-003 • E-mail: aabizaid@uol.com.br

Recebido em: 10/3/2008 • Aceito em: 15/5/2008 
Maia F, et al. Análise Angiográfica e Ultra-sonográfica do Stent Liberador de Novolimus para o Tratamento de Lesões Coronárias De Novo: Resultados do Estudo EXCELLA I. Rev Bras Cardiol Invas. 2008;16(2):139-143.

0 surgimento de stents farmacológicos representou importante marco na história da intervenção coronária percutânea, com sensível redução nos índices de reestenose coronária ${ }^{1-4}$. Após a experiência inicial com os stents farmacológicos de primeira geração (Cypher ${ }^{\circledR}$ Com sirolimus e $\operatorname{Taxus}^{\circledR}$ Com paclitaxel $\left.^{5,6}\right)$, uma série de novos fármacos imunossupressores, principalmente da família dos macrolídeos (zotarolimus $^{7}$, biolimus ${ }^{8}$, everolimus ${ }^{9,10}$, pimecrolimus ${ }^{11}$ e tacrolimus ${ }^{12}$ ), passou a ser testada com diferentes plataformas e polímeros biocompatíveis, visando ao desenvolvimento de stents farmacológicos mais seguros e com perfil de eficácia pelo menos equivalente ao dos atualmente em uso clínico.

Recentemente desenvolvido, o stent farmacológico Excella (Elixir Medical Corp. Sunnyvale, CA, Estados Unidos) combina uma plataforma de cobalto-cromo de hastes finas, um polímero biocompatível de metacrilato e o novolimus, um macrolídeo com propriedades e mecanismo de ação semelhantes ao sirolimus.

Este estudo objetivou avaliar, pela primeira vez em humanos, a exeqüibilidade, a eficácia e a segurança dessa nova geração de stents farmacológicos.

\section{MÉTODO}

O EXCELLA I é um estudo tipo first-in-man, nãorandomizado, realizado no Instituto Dante Pazzanese de Cardiologia, no mês de maio de 2007. Nesse registro, foram incluídos pacientes portadores de lesões coronárias de novo, em artérias com diâmetro de referência entre $3,0 \mathrm{~mm}$ e $3,5 \mathrm{~mm}$, extensão da lesão $\leq 14 \mathrm{~mm}$ e que apresentassem angina estável ou instável ou fossem portadores de isquemia silenciosa. Foram excluídos os pacientes com infarto agudo do miocárdio $<72$ horas, lesões situadas em bifurcações com ramo lateral $>1,5 \mathrm{~mm}$, lesões em óstio coronário, lesões em tronco de artéria coronária esquerda e lesões em artérias muito calcificadas e/ou tortuosas à angiografia. Também foram excluídos indivíduos com disfunção ventricular grave (fração de ejeção < 25\%) e insuficiência renal (creatinina $>1,8 \mathrm{mg} / \mathrm{dl}$ ).

\section{DESCRIÇÃO DO STENT EXCELLA}

\section{Plataforma}

Trata-se de um stent de cobalto-cromo, com oito células, balão-expansível, de hastes ultrafinas (0,0032" ou $82 \mu \mathrm{m})$, montado em cateter-balão semicomplacente.

\section{Polímero}

O polímero é de metacrilato, durável, mas com propriedades biocompatíveis, e que tem como diferencial sua reduzida espessura $(\sim 3 \mu \mathrm{m})$. Esse polímero, já utilizado em outros stents farmacológicos, permite a total liberação do novolimus no período de quatro a seis semanas.

\section{Fármaco antiproliferativo}

O novolimus, como o sirolimus, é um macrolídeo capaz de formar um complexo imunossupressor que impede a ação do mTor, inibindo a progressão da fase G1 para S do ciclo celular. Esse mecanismo já foi descrito previamente em detalhes ${ }^{1}$. A dose do novolimus utilizada foi de $5 \mathrm{mcg} / \mathrm{mm}$ de stent.

\section{Procedimento}

No presente estudo, estavam disponíveis stents com diâmetros de 3,0 mm e 3,5 mm e extensão de 18 $\mathrm{mm}$. As lesões foram pré-dilatadas com cateter-balão e todos os pacientes encontravam-se em uso de terapia antiagregante plaquetária dupla no momento da intervenção coronária. O pré-tratamento com ácido acetilsalicílico (AAS) (200 mg) e clopidogrel (300 mg) foi realizado em todos os pacientes, 24 horas antes do procedimento. Após a intervenção, todos os pacientes foram orientados a manter o uso de AAS indefinidamente e de clopidogrel, por 12 meses.

\section{Angiografia coronária quantitativa}

A angiografia coronária quantitativa $(\mathrm{ACQ})$ do vasoalvo foi realizada pré e pós-procedimento, após administração de $100 \mu \mathrm{g}$ a $200 \mu \mathrm{g}$ de nitroglicerina intracoronária, em pelo menos duas projeções ortogonais. As mesmas projeções foram repetidas na avaliação angiográfica aos quatro meses de evolução. As imagens foram armazenadas em formato digital e analisadas utilizando-se sistema de detecção automática de bordas (QCA-CMS, Medis Medical Imaging System, Nuenen, Holanda).

Análise morfológica da lesão foi feita de acordo com os critérios padrão e sua complexidade foi definida de acordo com o sistema de classificação modificado do American College of Cardiology/American Heart Association.

A ponta do cateter-guia, totalmente preenchida por contraste, foi utilizada para calibração.

A análise quantitativa incluía a avaliação dos seguintes parâmetros: 1) diâmetro de referência do vaso; 2) diâmetro luminal mínimo (DLM); 3) extensão da lesão; 4) porcentual do diâmetro de estenose (diâmetro de referência - DLM/diâmetro de referência x 100); 5) ganho agudo (DLM pós-procedimento - DLM préprocedimento); e 6) perda tardia (DLM pós-procedimento - DLM tardio).

A análise quantitativa foi realizada intra-stent e intra-segmento (stent acrescido dos $5 \mathrm{~mm}$ proximais e distais a suas bordas). Reestenose binária foi definida como diâmetro de estenose $\geq 50 \%$

\section{Análise do ultra-som intracoronário}

As imagens do ultra-som intracoronário (USIC) pósprocedimento e aos quatro meses de seguimento foram adquiridas utilizando-se transdutor de elemento 
Maia F, et al. Análise Angiográfica e Ultra-sonográfica do Stent Liberador de Novolimus para o Tratamento de Lesões Coronárias De Novo: Resultados do Estudo EXCELLA I. Rev Bras Cardiol Invas. 2008;16(2):139-143.

único, rotacional, com freqüência de $40 \mathrm{MHz}$, envolto em uma bainha com perfil 2,6 French, com recuo motorizado em sistema de tração automática à velocidade de $0,5 \mathrm{~mm} / \mathrm{s}$ e conectado a um scanner comercial (Galaxy 2, Boston Scientific Corp., Nattick, MA, Estados Unidos).

As imagens foram digitalizadas para análise quantitativa off-line, de acordo com os critérios do American College of Cardiology Clinical Expert Consensus Document on IVUS.

Para análise volumétrica, foi realizada a reconstrução tridimensional das imagens do USIC utilizandose um programa de planimetria computadorizada comercialmente disponível (EchoPlaque 2, INDEC Systems Inc., Mountain View, CA, Estados Unidos). As áreas da luz, do stent e do vaso (membrana elástica externa) foram determinadas, a cada milímetro, por planimetria computadorizada. A área da hiperplasia neo-intimal foi calculada como a diferença entre a área do stent e a área da luz. A partir de então, os volumes (da luz, do stent e do vaso) foram calculados pela regra de Simpson. O porcentual de obstrução intra-stent foi calculado como a razão entre o volume da hiperplasia intimal e o volume do stent x 100. Aposição incompleta do stent foi definida como a separação de pelo menos uma haste do stent da parede vascular, com presença de fluxo por trás das hastes, sem envolver uma área de bifurcação com outro ramo. Aposição incompleta era ainda classificada como persistente (presente após o procedimento e no seguimento), resolvida (presente após o procedimento, mas ausente no seguimento) e adquirida (ausente após o procedimento e presente no seguimento).

\section{Desfecho primário}

Perda luminal tardia intra-stent, quatro meses após o procedimento, avaliada pela ACQ.

\section{Desfechos secundários}

- Desfechos clínicos:

1. Eventos adversos cardíacos maiores (ECAM) até dois anos de seguimento clínico. ECAM foram definidos como óbito, infarto agudo do miocárdio com e sem elevação do segmento ST ou nova revascularização por isquemia relacionada à lesão-alvo, por cirurgia de revascularização miocárdica ou nova angioplastia coronária.

\section{- Desfechos angiográficos:}

1. Reestenose binária ( $\geq 50 \%$ ) aos quatro meses.

2. DLM e porcentagem do diâmetro de estenose (DS) aos quatro meses.

\section{- Desfechos ultra-sonográficos:}

1. Volume de hiperplasia intra-stent aos quatro meses.

2. Porcentual de obstrução do stent aos quatro meses.

\section{RESULTADOS}

O estudo incluiu 15 pacientes, com média de idade de 60,8 \pm 10,7 anos. A maioria dos pacientes $(87 \%)$ era portadora de doença arterial coronária estável (isquemia silenciosa ou angina estável). Chama atenção a alta prevalência de pacientes diabéticos (47\%) e com história de infarto do miocárdio prévio (73\%), conferindo a essa população perfil de risco cardiovascular pelo menos moderado (Tabela 1 ).

TABELA 1

\section{Características clínicas, angiográficas e do procedimento}

\begin{tabular}{|c|c|}
\hline Características & Pacientes $(n=15)$ \\
\hline Média de idade, anos & $60,8 \pm 10,7$ \\
\hline Sexo feminino & $10(67 \%)$ \\
\hline Hipertensão arterial & $15(100 \%)$ \\
\hline Dislipidemia & $12(80 \%)$ \\
\hline Diabetes melito & $7(47 \%)$ \\
\hline Tabagismo & $2(13 \%)$ \\
\hline História familiar de DAC & $7(47 \%)$ \\
\hline IAM prévio & $11(73 \%)$ \\
\hline ICP prévia & $1(6 \%)$ \\
\hline \multicolumn{2}{|l|}{ Apresentação clínica } \\
\hline Angina estável/isquemia silenciosa & $13(87 \%)$ \\
\hline Angina instável & $2(13 \%)$ \\
\hline \multicolumn{2}{|l|}{ Vaso tratado } \\
\hline DA & $7(48 \%)$ \\
\hline $\mathrm{Cx}$ & $4(26 \%)$ \\
\hline CD & $4(26 \%)$ \\
\hline Pré-dilatação & $15(100 \%)$ \\
\hline Pós-dilatação & $11(73 \%)$ \\
\hline Número de stents por lesão & 1,06 \\
\hline Pressão média de liberação final, atm & $16,5 \pm 2,8$ \\
\hline \multicolumn{2}{|l|}{ ACQ pré-procedimento } \\
\hline Diâmetro de referência média & \\
\hline do vaso, $\mathrm{mm}$ & $2,7 \pm 0,4$ \\
\hline Extensão média da lesão, mm & $8,7 \pm 3,7$ \\
\hline DLM, mm & $1,0 \pm 0,3$ \\
\hline DS, \% & $62,5 \pm 8,6$ \\
\hline \multicolumn{2}{|l|}{ ACQ pós-procedimento } \\
\hline DLM intra-stent, mm & $2,5 \pm 0,3$ \\
\hline DLM segmento, mm & $2,2 \pm 0,4$ \\
\hline DS intra-stent, mm & $7,4 \pm 9,6$ \\
\hline DS segmento, mm & $20,8 \pm 1,1$ \\
\hline Ganho agudo no segmento, mm & $1,5 \pm 0,3$ \\
\hline Sucesso angiográfico & $15(100 \%)$ \\
\hline Sucesso do procedimento & $15(100 \%)$ \\
\hline
\end{tabular}

$\mathrm{ACQ}$ = angiografia coronária quantitativa; $\mathrm{CD}=$ artéria coronária direita; $\mathrm{Cx}=$ artéria circunflexa; DA = artéria descendente anterior; $\mathrm{DAC}=$ doença arterial coronária; $\mathrm{DLM}=$ diâmetro luminal mínimo; $\mathrm{DS}$ = diâmetro de estenose; IAM = infarto agudo do miocárdio; ICP = intervenção coronária percutânea. 
Maia F, et al. Análise Angiográfica e Ultra-sonográfica do Stent Liberador de Novolimus para o Tratamento de Lesões Coronárias De Novo: Resultados do Estudo EXCELLA I. Rev Bras Cardiol Invas. 2008;16(2):139-143.

Todos os stents foram liberados com sucesso e os pacientes receberam alta hospitalar 24 horas após o procedimento, sem quaisquer intercorrências. As enzimas cardíacas (CK total e CK-MB atividade), dosadas 12 e 24 horas após o procedimento, não demonstraram elevações significativas.

Dados da análise tanto angiográfica como volumétrica (ACQ e USIC) dos pacientes são apresentados nas Tabelas 2 e 3. Não foi observado nenhum caso de reestenose binária $>50 \%$, e a perda tardia, tanto intra-stent como no segmento, foi mínima. Também não foram constatados casos de aposição incompleta persistente, resolvida ou adquirida do stent. A Figura 1 ilustra dois exemplos de pacientes incluídos no presente estudo.

Todos os pacientes completaram o seguimento clínico aos seis meses, sem a ocorrência de ECAM ou trombose de stent.

\section{DISCUSSÃO}

O principal achado deste estudo pioneiro é que o uso do stent Excella não só é factível como parece correlacionar-se com perfil de eficácia satisfatório, com a constatação da marcante redução do tecido neointimal tanto à ACQ quanto ao USIC.
Embora utilize um polímero durável, o stent Excella, por conter baixa concentração de novolimus, necessita de quantidade menor de polímero para liberar o fármaco antiproliferativo no período de tempo programado.

Como outros atrativos, esse stent possui moderna plataforma de cobalto-cromo, de hastes muito finas, mas com boa radiopacidade, tendo demonstrado excelente navegabilidade em estudos pré-clínicos ${ }^{13}$.

Os resultados angiográficos e ultra-sonográficos preliminares deste estudo colocam o stent liberador de novolimus em excelente patamar de efetividade, com desempenho marcadamente superior ao dos stents não-farmacológicos ${ }^{1-3}$. Quando comparado aos stents farmacológicos aprovados pelo Food and Drug Administration (FDA), demonstra melhor resultado que os stents Endeavor ${ }^{\circledR 7}$ e Taxus $^{\circledR 5,6}$ e resultado equivalente ao dos stents Cypher ${ }^{\circledR 1-4}$ e PromusT/Xience ${ }^{9}$ em cenários de baixa a média complexidade clínica e angiográfica (Figura 2).

\section{Limitações}

Este estudo abrange uma amostra pequena de pacientes $(n=15)$, com seguimento angiográfico de quatro meses e seguimento clínico de seis meses. Resultados angiográficos aos oito meses e clínicos aos

TABELA 2

Angiografia coronária quantitativa

\begin{tabular}{lccc}
\hline & Pós-procedimento $(\mathbf{n}=\mathbf{1 5})$ & Quatro meses $(\mathbf{n}=\mathbf{1 5})$ & Valor de $\mathbf{p}$ \\
\hline DLM intra-stent, $\mathrm{mm}$ & $2,5 \pm 0,3$ & $2,3 \pm 0,4$ & 0,46 \\
DLM segmento, mm & $2,2 \pm 0,4$ & $2,1 \pm 0,5$ & 0,57 \\
DS intra-stent, $\%$ & $7,4 \pm 9,6$ & $12,5 \pm 13,1$ & 0,24 \\
DS segmento, $\%$ & $20,8 \pm 11$ & $22,2 \pm 0,3$ & 0,44 \\
Perda luminal tardia intra-stent, mm & $\mathrm{N} / \mathrm{A}$ & $0,15 \pm 0,29$ & $\mathrm{~N} / \mathrm{A}$ \\
Perda luminal tardia segmento, $\mathrm{mm}$ & $\mathrm{N} / \mathrm{A}$ & $0,07 \pm 0,30$ & $\mathrm{~N} / \mathrm{A}$ \\
\hline
\end{tabular}

$\mathrm{ACQ}$ = angiografia coronária quantitativa; DLM = diâmetro luminal mínimo; $\mathrm{DS}$ = diâmetro de estenose; $\mathrm{n}=$ número de pacientes; $\mathrm{N} / \mathrm{A}=$ não avaliado.

TABELA 3 Ultra-som intracoronário

\begin{tabular}{lccc}
\hline & Pós-procedimento $\left(\mathbf{n}=\mathbf{1 4}^{*}\right)$ & Quatro meses $(\mathbf{n}=\mathbf{1 4}$ ) & Valor de $\mathbf{p}$ \\
\hline Volume do vaso, $\mathrm{mm}^{3}$ & $251,5 \pm 74,3$ & $264,7 \pm 81,2$ & 0,9 \\
Volume do stent, $\mathrm{mm}^{3}$ & $131,2 \pm 38,4$ & $137,8 \pm 38,0$ & 0,8 \\
Volume da luz, $\mathrm{mm}^{3}$ & $131,0 \pm 38,4$ & $134,8 \pm 38,0$ & 0,9 \\
Volume neo-intimal, $\mathrm{mm}^{3} / \mathrm{mm}$ & $\mathrm{N} / \mathrm{A}$ & $0,17 \pm 0,14$ & $\mathrm{~N} / \mathrm{A}$ \\
Volume de obstruçãointra-stent, $\%$ & $\mathrm{~N} / \mathrm{A}$ & $2,6 \pm 2,6$ & $\mathrm{~N} / \mathrm{A}$ \\
\hline
\end{tabular}

* Não foi possível a realização do ultra-som intracoronário em um paciente. Números são médias de desvio padrão.

$\mathrm{n}=$ número de pacientes; $\mathrm{N} / \mathrm{A}=$ não avaliado. 


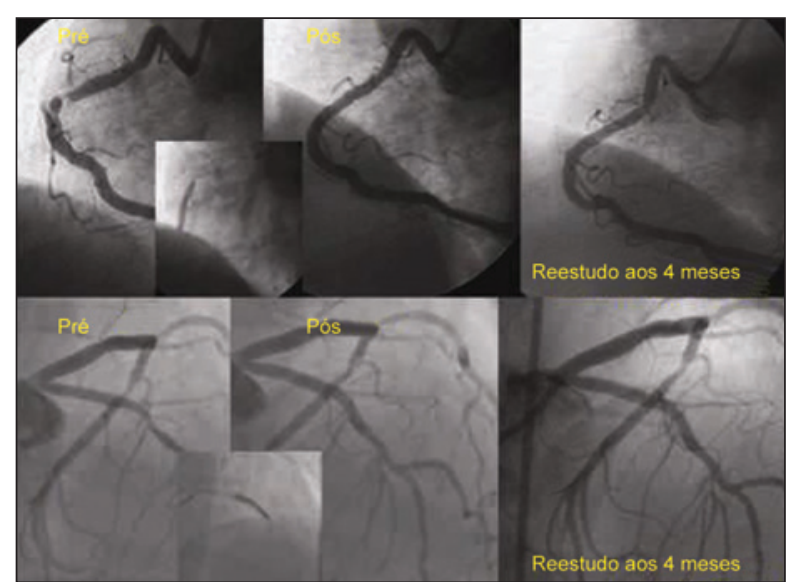

Figura 1 - Dois exemplos da série inicial de 15 pacientes tratados no Instituto Dante Pazzanese de Cardiologia. Em ambos é marcante a supressão da hiperplasia neo-intimal no interior do stent Excella.

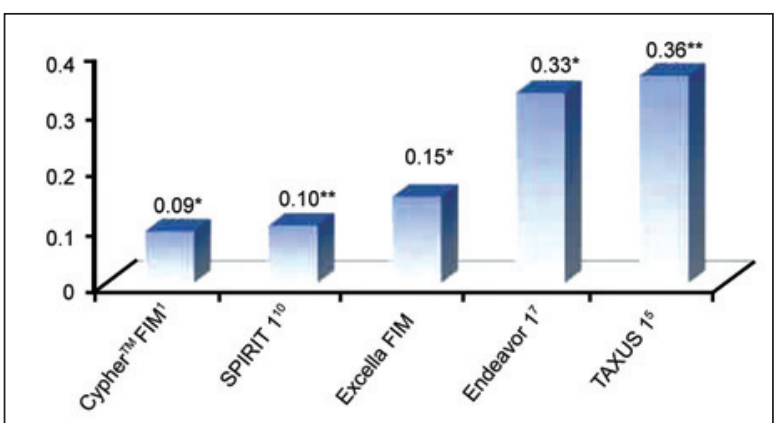

Figura 2 - Perda tardia do stent Excella comparada à perda tardia reportada dos stents farmacológicos (SF) aprovados pelo Food and Drug Administration (FDA). Quando comparado aos SF testados em cenário de complexidade similar, o stent Excella demonstrou eficácia ligeiramente superior à dos stents Endeavor ${ }^{\circledR}$ e Taxus $^{\circledR}$, e equivalente à dos stents Cypher $^{\circledR}$ e PromusT/Xience.

* Reestudo angiográfico aos quatro meses.

** Reestudo angiográfico aos seis meses.

24 meses serão necessários para confirmar esses resultados iniciais.

\section{CONCLUSÃO}

O stent Excella demonstrou ser seguro e eficaz na inibição da hiperplasia neo-intimal, quatro meses após o implante em lesões coronárias de novo. Tais achados necessitam ser confirmados por estudos multicêntricos, randomizados, em populações com perfil de maior complexidade tanto clínica como angiográfica.

\section{REFERÊNCIAS BIBLIOGRÁFICAS}

1. Sousa JE, Costa MA, Abizaid A, Abizaid AS, Feres F, Pinto $I M$, et al. Lack of neointimal proliferation after implantation of sirolimus-coated stents in humans coronary arteries: a quantitative coronary angiography and three-dimensional intravascular ultrasound study. Circulation. 2001;103(2): $192-5$

2. Morice MC, Serruys PW, Sousa JE, Fajadet J, Ban Hayashi $\mathrm{E}$, Perin $\mathrm{M}$, et al. A randomized comparison of a sirolimuseluting stent with a standard stent for coronary revascularization. N Engl J Med. 2002;346(23):1773-80.

3. Moses JW, Leon MB, Popma JJ, Fitzgerald PJ, Holmes DR, $\mathrm{O}^{\prime}$ Shaughnessy C, et al. Sirolimus-eluting stents versus standard stents in patients with stenosis in a native coronary artery. N Engl J Med. 2003;349(14):1315-23.

4. Abizaid AAC, Sousa AGMR, Abizaid ACLS, Costa MA, Langenhove GV, Kozuma K, et al. Sustained suppression of neointimal proliferation late (1 year) after implantation of sirolimuseluting BX-velocity stent. Circulation. 2001;104(Suppl II):464.

5. Grube E, Silber S, Hauptmann KE, Mueller R, Buellesfeld L, Gerckens $U$, et al. TAXUS I: six- and twelve-month results from a randomized, double-blind trial on a slow-release paclitaxel-eluting stent for de novo coronary lesions. Circulation. 2003;107(1):38-42.

6. Colombo A, Drzewiecki J, Banning A, Grube E, Hauptmann K, Silber S, et al. Randomized study to assess the effectiveness of slow- and moderate-release polymer-based paclitaxeleluting stents for coronary artery lesions. Circulation. 2003; 108(7):788-94

7. Meredith IT, Ormiston J, Whitbourn R. First-in-human study of the Endeavor ABT-578-eluting phosphorylcholine-encapsulated stent system in de novo native coronary artery lesions: ENDEAVOR I Trial. Eurolntervention. 2005;1:157-64.

8. Costa RA, Lansky AJ, Abizaid A, Müeller R, Tsuchiya Y, Mori K, et al. Angiographic results of the first human experience with the Biolimus A9 drug-eluting stent for de novo coronary lesions. Am J Cardiol. 2006;98(4):443-6.

9. Serruys PW, Ong ATL, Piek JJ. A randomized comparison of a durable polymer Everolimus-eluting stent with a bare metal coronary stent: The SPIRIT first trial. Eurolntervention. 2005; 1(1):58-65.

10. Grube E, Sonoda S, Ikeno F, Honda Y, Kar S, Chan C, et al. Six- and twelve-month results from first human experience using everolimus-eluting stents with bioabsorbable polymer. Circulation. 2004;109(18):2168-71.

11. Abizaid A. Avantec pimecrolimus eluting stent. In: Transcatheter Cardiovascular Therapeutics Symposium; October 17-21,2005; Washington, DC.

12. Morice MC, Bestehorn HP, Carrie D, Macaya C, Aengevaeren W, Wijns W, et al. Direct stenting of de novo coronary stenoses with tacrolimus-eluting versus carbon-coated carbostents: the randomized JUPITER II trial. Eurolntervention. 2006;2(1):45-52.

13. Hagemeister J, Baer FM, Schwinger RH, Höpp HW. Compliance of a cobalt chromium coronary stent alloy: the COVIS trial. Curr Control Trials Cardiovasc Med. 2005;6:17. 